\title{
Rate theory of acceleration of defect annealing driven by discrete breathers
}

\author{
Vladimir I. Dubinko, Juan F. R. Archilla, Sergey V. Dmitriev, and \\ Vladimir Hizhnyakov

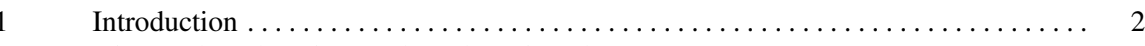 \\ 2 Discrete breathers in metals and semiconductors $\ldots \ldots \ldots \ldots \ldots \ldots \ldots \ldots \ldots \ldots \ldots \ldots$ \\ 3 DB excitation under thermal equilibrium and external driving $\ldots \ldots \ldots \ldots \ldots \ldots$ \\ 4 Amplification of Sb-vacancy annealing rate in germanium by DBs . . . . . . . . . 12

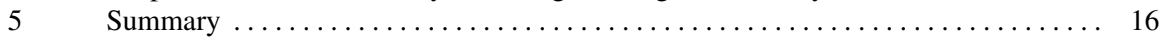

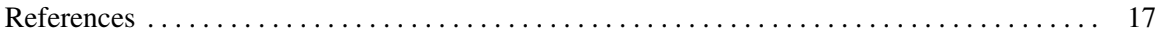

\begin{abstract}
Novel mechanisms of defect annealing in solids are discussed, which are based on the large amplitude anharmonic lattice vibrations, a.k.a. intrinsic localized modes or discrete breathers (DBs). A model for amplification of defect annealing rate in Ge by low energy plasma-generated DBs is proposed, in which, based on recent atomistic modelling, it is assumed that DBs can excite atoms around defects rather strongly, giving them energy $\gg k_{B} T$ for $\sim 100$ oscillation periods. This is shown to result in the amplification of the annealing rates proportional to the DB flux, i.e. to the flux of ions (or energetic atoms) impinging at the Ge surface from inductively coupled plasma (ICP).
\end{abstract}

Key words: Anharmonic lattice vibrations, discrete breathers, intrinsic localized modes, defect annealing

V.I. Dubinko

NSC Kharkov Institute of Physics and Technology, Akademicheskya Str. 1, Kharkov 61108, Ukraine, e-mail: vdubinko@mail.ru

J.F.R. Archilla

Group of Nonlinear Physics, Universidad de Sevilla, ETSI Informática, Avda. Reina Mercedes s/n 41011, Seville, Spain, e-mail: archilla@us.es

S.V. Dmitriev

Institute for Metals Superplasticity Problems, RAS, Khalturin Str. 39, Ufa 450001, Russia

National Research Tomsk State University, Lenin Prospekt 36, Tomsk 634050, Russia, e-mail: dmitriev.sergey.v@gmail.com

V. Hizhnyakov

Institute of Physics, University of Tartu, Riia 142, EE-51014 Tartu, Estonia, e-mail: hizh@fi.tartu.ee 


\section{Introduction}

A defect lying in the band gap with energy $>0.1 \mathrm{eV}$ from either band edge is termed deep. As known from the studies of properties of defects in Ge $[1,2,4,5,30]$, Ar ions arriving at a semiconductor surface with very low energy $(2-8 \mathrm{eV})$ are annihilating defects deep inside the semiconductor. Several different defects were removed or modified in Sb-doped germanium, of which the $E$-center has the highest concentration, as described in details in Ref. [1,2]. Novel mechanisms of defect annealing in solids are discussed in this work, which are based on the large amplitude anharmonic lattice vibrations, a.k.a. intrinsic localized modes (ILMs) or discrete breathers (DBs). The article is organized as follows. In Sect. 2, a short review on DB properties in metals and semiconductors is presented based on the results of molecular dynamics (MD) simulations using realistic many-body interatomic potentials. In Sect. 3, a rate theory of DB excitation under thermal heating and under non-equilibrium gas loading conditions is developed. In Sect. 4, a model for amplification of defect annealing rate in Ge by plasma-generated DBs is proposed and compared with experimental data. The results are summarized in Sect. 5.

\section{Discrete breathers in metals and semiconductors}

DBs are spatially localized large-amplitude vibrational modes in lattices that exhibit strong anharmonicity $[14,20,33,35]$. They have been identified as exact solutions to a number of model nonlinear systems possessing translational symmetry [14] and successfully observed experimentally in various physical systems [14,29]. Presently the interest of researchers has shifted to the study of the role of DBs in solid state physics and their impact on the physical properties of materials $[9,10,12,13,29,36]$. Until recently the evidence for the DB existence provided by direct atomistic simulations, e.g. MD, was restricted mainly to one and two-dimensional networks of coupled nonlinear oscillators employing oversimplified pairwise inter-particle potentials $[14,20,33]$. Studies of the DBs in three-dimensional systems by means of MD simulations using realistic interatomic potentials include ionic crystals with $\mathrm{NaCl}$ structure [21, 25], graphene [6, 23, 27], graphane [28], semiconductors [37], pure metals $[15,18,32,36]$, and ordered alloys [31]. For the first time the density functional theory (DFT) was applied to the study of DBs, using graphane as an example [7].

DBs have very long lifetime because their frequencies lie outside the phonon band. Monatomic crystals like pure metals and semiconductors such as $\mathrm{Si}$ and $\mathrm{Ge}$ do not possess gaps in the phonon spectrum, while crystals with complex structure often have such gaps, for example, diatomic alkali halide crystals and ordered alloys with a large difference in the atomic mass of the components. For the crystals possessing a gap in the phonon spectrum the so-called gap DBs with frequencies within the gap can be excited. This case will not be discussed here and in the following we focus on the DBs having frequencies above the phonon band. 


\subsection{Metals}

In the work by Kiselev et al. [24] it has been demonstrated that 1D chain of particles interacting with the nearest neighbors via classical pairwise potentials such as Toda, Lennadrd-Jones or Morse cannot support DBs with frequencies above the phonon band. They were able to excite only gap DBs with frequencies lying within the gap of the phonon spectrum by considering diatomic chains. In line with the results of this work, it was accepted for a long time that the softening of atomic bonds with increasing vibrational amplitude is a general property of crystals, which means that the oscillation frequency decreases with increasing amplitude. Therefore DBs with frequencies above the top phonon frequency were unexpected.

However, in 2011, Haas et al. [15] have demonstrated by MD simulations using realistic many-body interatomic potentials that DBs with frequencies above the phonon spectrum can be excited in fcc $\mathrm{Ni}$ as well as in bec $\mathrm{Nb}$ and $\mathrm{Fe}[15,18]$. Similar results were obtained for bcc Fe, V, and W [32].

The point is that the realistic interatomic potentials, including Lennard-Jones and Morse, have an inflection point meaning that they are composed of the hard core and the soft tail. This is typical for interatomic bonds of any complexity, including many-body potentials. Physically the soft tail is due to the interaction of the outer electron shells of the atoms, while the hard core originates from the strong repulsive forces between nuclei and also from the Pauli exclusion principle for inner electrons (fermions) that cannot occupy the same quantum state simultaneously. It is thus important which part of the interatomic potential (hard or soft) contributes more to the dynamics of the system. As it was shown in [24], the asymmetry of the interatomic potentials results in the thermal expansion effect when larger vibrational amplitudes, at zero pressure, cause the larger equilibrium interatomic distance and hence, a larger contribution from the soft tail. If thermal expansion is suppressed somehow, then the hard core manifests itself. To demonstrate this let us consider the Morse chain of unit mass particles whose dynamics is described by the following equations of motion

$$
\ddot{u}_{n}=U^{\prime}\left(h+u_{n+1}-u_{n}\right)-U^{\prime}\left(h+u_{n}-u_{n-1}\right),
$$

where $u_{n}(t)$ is the displacement of the $n$th particle from the lattice position, $h$ is the lattice spacing,

$$
U(r)=D\left(e^{-2 \alpha\left(r-r_{m}\right)}-2 e^{-\alpha\left(r-r_{m}\right)}\right),
$$

is the Morse potential, where $r$ is the distance between two atoms, $D, \alpha, r_{m}$ are the potential parameters. The function $U(r)$ has a minimum at $r=r_{m}$, the depth of the potential (the binding energy) is equal to $D$ and $\alpha$ defines the stiffness of the bond. We take $D=1, r_{m}=1$ and $\alpha=5$. For the considered case of the nearest-neighbor interactions the equilibrium interatomic distance is $h=r_{m}=1$.

In frame of the model given in Eqs. (1), (2) we study the dynamics of the staggered mode excited with the use of the following initial conditions

$$
u_{n}(0)=A \cos (\pi n)=(-1)^{n} A, \quad \dot{u}_{n}(0)=0,
$$


in the chain of $N$ particles ( $N$ is an even number) subjected to the periodic boundary conditions, $u_{n}(t)=u_{n+N}(t)$. Our aim is to find the frequency of the mode as the function of the mode amplitude $A$ for the two cases. Firstly the chain is allowed to expand, and for given $A>0$ the interatomic distance $h>1$ is such that the pressure $p=0$. In the second case the thermal expansion is suppressed by fixing $h=1$ for any $A$. In this case, of course, for $A>0$ one has $p>0$. The results for the two cases are shown in Fig. 1 (a) and (b), respectively. In (a) the frequency of the mode decreases with $A$, while in (b) the opposite takes place.

In the numerical experiments by Haas et al. [15] is was found that the DBs in pure metals are extended along a close-packed atomic row. The atoms surrounding the atomic row where DB is excited create the effective periodic on-site potential that suppresses the thermal expansion of the row and that is why the DB frequency increases with increasing amplitude. The on-site potential was not introduced in the 1D model by Kiselev et al. [24] and, naturally, thermal expansion did not allow for the existence of DBs with frequencies above the phonon band.

Notably, the excitation energy of DBs in metals can be relatively small (fractions of eV) as compared to the formation energy of a stable Frenkel pair (several eV). Moreover, it has been shown that DBs in pure metals are highly mobile and hence they can efficiently transfer energy and momentum over large distances along closepacked crystallographic directions $[18,32,36]$. Recently, a theoretical background has been proposed to ascribe the interaction of moving DBs (a.k.a 'quodons' - quasi-

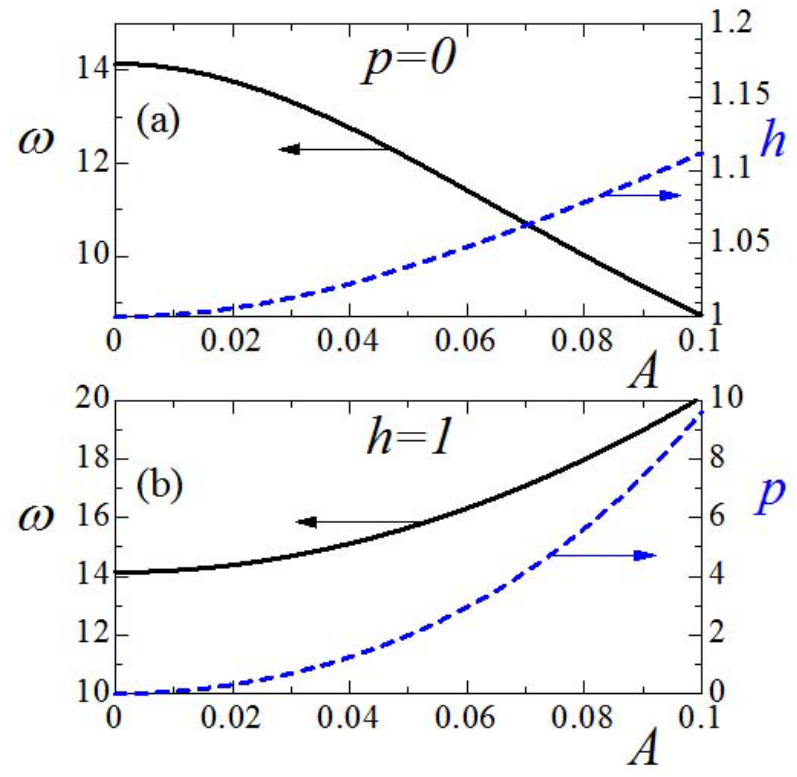

Fig. 1 Solid lines show frequency of the staggered mode (left ordinate) as the function of amplitude for the case of (a) $p=0$ and (b) $h=1$. Dashed lines show (a) $h$ and (b) $p$ (right ordinate) as the functions of $A$. The results for the 1D Morse lattice (1), (2) with the initial conditions (3). 
particles propagating along close-packed crystallographic directions) with defects in metals to explain the anomalously accelerated chemical reactions in metals subjected to irradiation. Russell and Eilbeck [34] have presented experimental evidence for the existence of quodons that propagate great distances in atomic-chain directions in crystals of muscovite, an insulating solid with a layered crystal structure. Specifically, when a crystal of muscovite was bombarded with alpha-particles at a given point at $300 \mathrm{~K}$, atoms were ejected from remote points on another face of the crystal, lying in atomic chain directions at more than $10^{7}$ unit cells distance from the site of bombardment. Irradiation may cause continuous generation of DBs inside materials due to external lattice excitation, thus 'pumping' a material with DB gas $[10,12]$.

In order to understand better the structure and properties of standing and moving DBs, consider the ways of their external excitation in Fe by MD simulations [36]. A standing DB can be excited by applying the initial displacements to the two adjacent atoms along the close-packed [111] direction with the opposite signs to initiate their anti-phase oscillations, as shown in Fig. 2(a). The initial displacements $\pm d_{0}$ determine the DB amplitude, frequency and, ultimately, its lifetime. DBs can be excited in a frequency band (1.0-1.4) $\times 10^{13} \mathrm{~Hz}$ just above the Debye frequency of bcc Fe, and DB frequency grows with increasing amplitude as expected for the hard type anharmonicity due to the major contribution from the hard core of the interatomic potential. Initial displacements larger than $\left|d_{0}\right|=0.45 \AA$ generate a chain of focusons, while displacements smaller than $\left|d_{0}\right|=0.27 \AA$ do not provide enough potential energy for the system to initiate a stable DB and the atomic oscillations decay quickly by losing its energy to phonons. The most stable DBs can survive up to 400 oscillations, as shown in Fig. 2(b), and ultimately decay in a stepwise quantum nature by generating bursts of phonons, as has been predicted by Hizhnyakov as early as in 1996 [17].

A moving DB can be excited by introducing certain asymmetry into the initial conditions. Particularly, the translational kinetic energy $E_{t r}$ can be given to the two central atoms of DB in the same direction along [111] atomic row. DB velocity ranges from 0.1 to 0.5 of the velocity of sound, while travel distances range from several dozens to several hundreds of the atomic spaces, depending on $d_{0}$ and $E_{t r}$ $[32,36]$. Figure 3(a) shows a DB passing the two neighboring atoms with indices 3415 and 3416. In the moving DB the two central atoms pulsate not exactly in antiphase but with a phase shift. In about 1 ps ( $\sim 10$ oscillations) the oscillations of these two atoms cease but they are resumed at the subsequent atoms along [111] atomic row. In this way, the DB moves at a speed of $2.14 \mathrm{~km} / \mathrm{s}$, i.e. about the half speed of sound in bcc Fe. The translational kinetic energy of the DB is about $0.54 \mathrm{eV}$, which is shared mainly among two core atoms, giving $0.27 \mathrm{eV}$ per atom, which is close to the initial kinetic energy of $E_{t r}=0.3 \mathrm{eV}$ given to the atoms to initiate the DB translational motion. The deviation of the potential energy of the atoms from the ground state during the passage of the DB is presented in Fig. 3(b). The maximal deviation of energy is of the order of $1 \mathrm{eV}$. Thus, a moving DB can be viewed as an atom-size localised excitation with local temperature above $1000 \mathrm{~K}$ propagating along the crystal at a subsonic speed. 

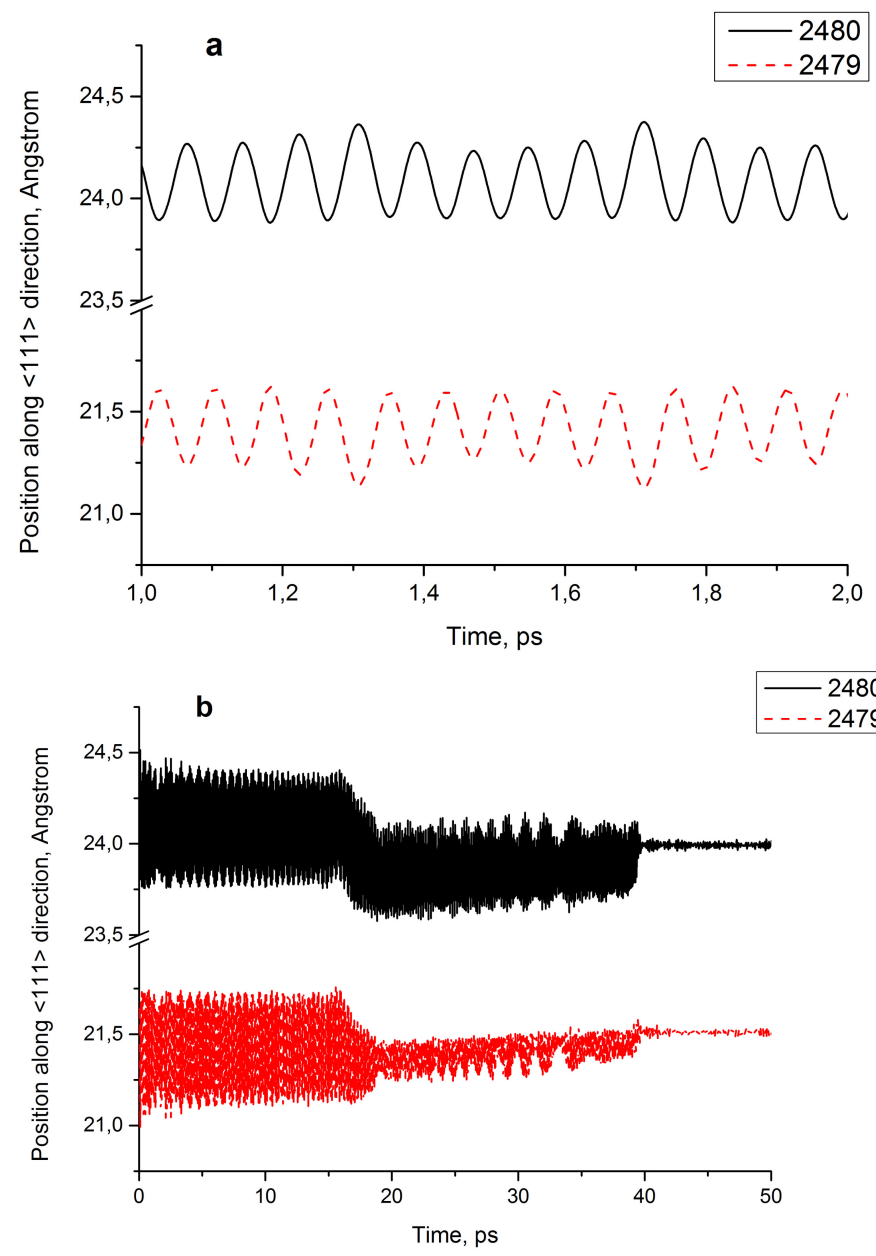

Fig. 2 Oscillation of $x$ coordinate of two neighbouring atoms, 2480 and 2479, in a [111] row in Fe in a standing DB excited with $d_{0}=0.325 \AA$ [36]. (a) Initial stage of DB evolution; (b) total lifespan of DB showing a stepwise quantum nature of its decay

\subsection{Semiconductors}

Similar to metals, semiconductors possess no gap in phonon spectrum and thus DBs may exist only if their frequency is positioned above the phonon spectrum $[15,37]$. Such high-frequency DBs may be realized in semiconductors due to the screening of the short-range covalent interaction by the conducting electrons. Voulgarakis et al. [37] investigated numerically existence and dynamical properties of DBs in crystalline silicon through the use of the Tersoff interatomic potential. They found a band of DBs with lifetime of at least $60 \mathrm{ps}$ in the spectral region 

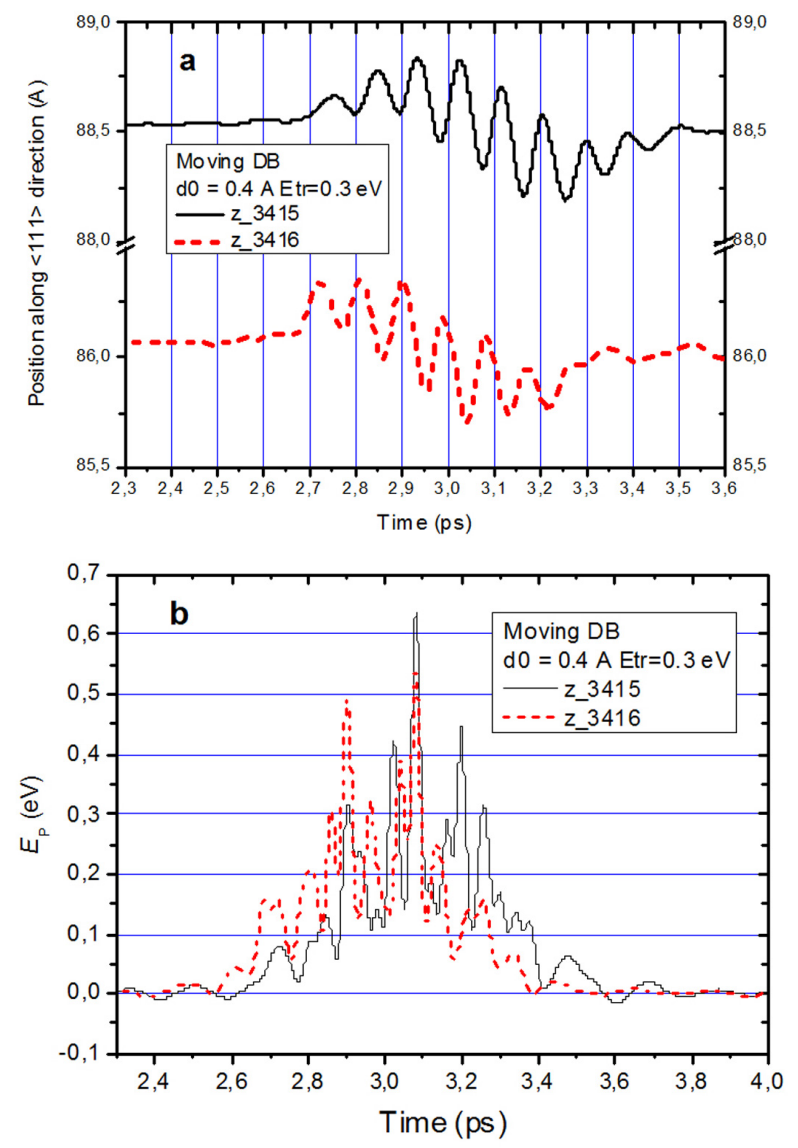

Fig. 3 (a) Oscillation of $x$ coordinate of two neighbouring atoms, 3415 and 3416 in a [111] row in Fe during the passage of a moving DB $\left(d_{0}=0.4 \AA, E_{t r}=0.3 \mathrm{eV}\right)$; (b) deviation of the potential energy of the atoms from the ground state during the passage of DB

$(1.643-1.733) \times 10^{13} \mathrm{~Hz}$, located just above the upper edge of the phonon band calculated at $1.607 \times 10^{13} \mathrm{~Hz}$. The localized modes extend to more than second neighbors and involve pair central-atom compressions in the range from $6.1 \%$ to $8.6 \%$ of the covalent bond length per atom. Finite temperature simulations showed that they remain robust to room temperatures or higher with a typical lifetime equal to 6 ps. Figure 4 shows DB generated in silicon modeled by the Tersoff potential [37]. It can be seen that the DB is very persistent and localized: its vibrational energy is mainly concentrated in the bond between two neighboring atoms oscillating in anti-phase mode.

Similar to silicon, germanium has a diamond crystal structure and readily produces DBs [19], as demonstrated in Fig. 5. As in Si, the DB's energy in Ge is concentrated in the central bond between two atoms oscillating in anti-phase mode. 

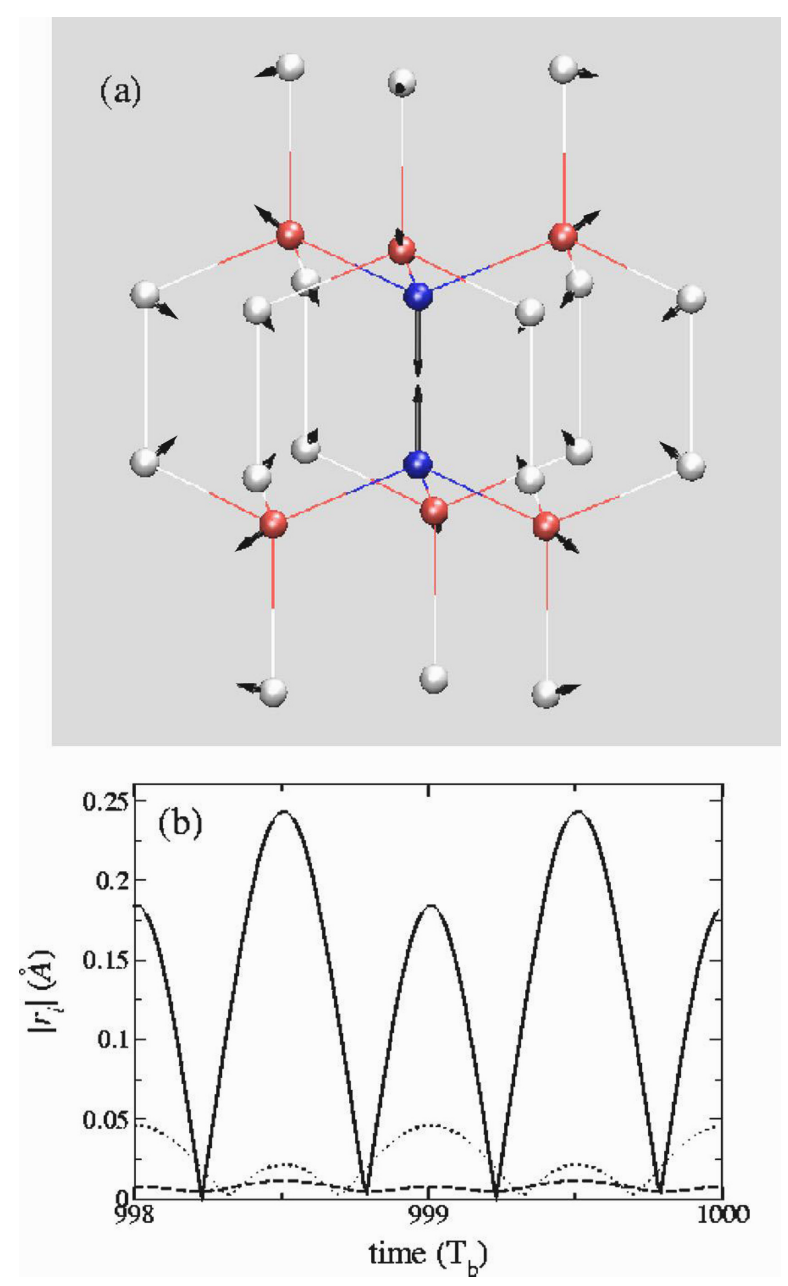

Fig. 4 (a) DB generation in silicon modeled by Tersoff potentials. The DB frequency is $1.733 \times$ $10^{13} \mathrm{~Hz}$, while vectors (magnified for visualization purposes) denote atomic displacements from equilibrium; only first (gray, red online) and second (white) neighbors to the central (black, blue online) two breather atoms are included. The displacement of the two central breather atoms is 0.18 A. (b) Time evolution of the silicon DB after 998 breather periods. The absolute value of the displacements from equilibrium along the direction of motion of each atom is plotted. The coordinated oscillations of central (solid), first (dotted), and second (dashed) neighbor atoms are indicated. Reproduced with permission from Voulgarakis, N., Hadjisavvas, G., Kelires, P., Tsironis, G.: Computational investigation of intrinsic localization in crystalline Si. Phys. Rev. B 69, 113,201 (2004). Copyright (2004) American Physical Society

This means that potential barriers for chemical reactions in the vicinity of an DB may be subjected to persistent periodic oscillations, which has been shown to result in a strong amplification of the reaction rates [13]. In the next section we consider the ways of DB excitation in thermal equilibrium and under external driving. 


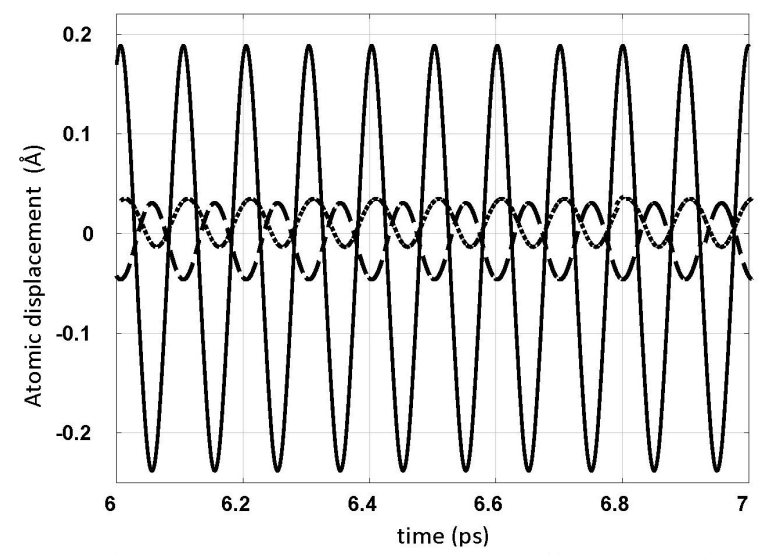

Fig. 5 DB generated in germanium modeled by the Tersoff potential. Displacement of one of the two central atoms is shown with a solid line and of the first neighbor by dashed (along [111] axis) and dotted (perpendicular to [111] axis) lines. See Ref. [19]
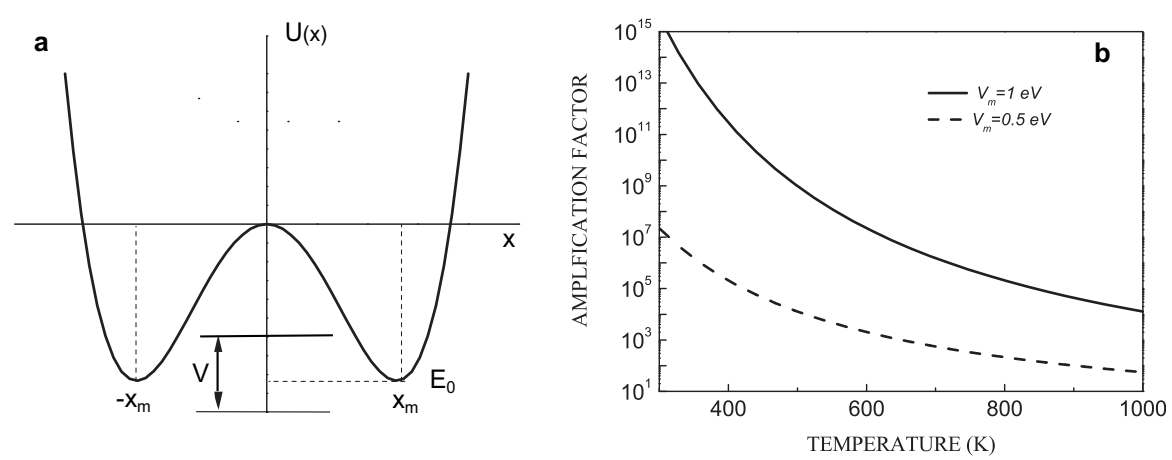

Fig. 6 (a) Sketch of the double-well potential landscape with minima located at $\pm x_{m}$. These are stable states before and after reaction, separated by a potential "barrier" with the height changing periodically or stochastically within the $V$ band. (b) Amplification factor, $I_{0}\left(V / k_{B} T\right)$, for the average escape rate of a thermalized Brownian particle from a periodically modulated potential barrier at different temperatures and modulation amplitudes $V$. Reproduced with permission from V.I. Dubinko, P.A. Selyshchev and J.F.R. Archilla: Reaction-rate theory with account of the crystal anharmonicity Phys. Rev. E 83041124 (2011). Copyright (2011) American Physical Society.

\section{DB excitation under thermal equilibrium and external driving}

In this section, for the convenience of the reader, we repeat the main points of the chemical reaction rate theory that takes into account the effect of DBs, following the earlier works $[3,11,13]$.

The rate equation for the concentration of DBs with energy $E, C_{D B}(E, t)$ can be written as follows [13] 


$$
\frac{\partial C_{D B}(E, t)}{\partial t}=K_{D B}(E)-\frac{C_{D B}(E, t)}{\tau_{D B}(E)},
$$

where $K_{D B}(E)$ is the rate of creation of DBs with energy $E>E_{\min }$ and $\tau_{D B}(E)$ is the DB lifetime. It has an obvious steady-state solution $\left(\partial C_{D B}(E, t) / \partial t=0\right)$ :

$$
C_{D B}(E)=K_{D B}(E) \tau_{D B}(E) .
$$

In the following sections we will consider the breather formation by thermal activation and then extend the model to non-equilibrium systems with external driving.

\subsection{Thermal activation}

The exponential dependence of the concentration of high-energy light atoms on temperature in the MD simulations [22] gives evidence in favor of their thermal activation at a rate given by a typical Arrhenius law [33]

$$
K_{D B}(E, T)=\omega_{D B} \exp \left(-\frac{E}{k_{B} T}\right),
$$

where $\omega_{D B}$ is the attempt frequency that should be close to the DB frequency. The breather lifetime has been proposed in [33] to be determined by a phenomenological law based on fairly general principles: (i) DBs in two and three dimensions have a minimum energy $E_{\min }$, (ii) The lifetime of a breather grows with its energy as $\tau_{D B}=\tau_{D B}^{0}\left(E / E_{\min }-1\right)^{z}$, with $z$ and $\tau_{D B}^{0}$ being constants, whence it follows that under thermal equilibrium, the DB energy distribution function $C_{D B}(E, T)$ and the mean number of breathers per site $n_{D B}(T)$ are given by

$$
\begin{gathered}
C_{D B}(E, T)=\omega_{D B} \tau_{D B} \exp \left(-\frac{E}{k_{B} T}\right), \\
n_{D B}(T)=\int_{E_{\min }}^{E_{\max }} C_{D B}(E, T) d E=\omega_{D B} \tau_{D B}^{0} \frac{\exp \left(-E_{\min } / k_{B} T\right)}{\left(E_{\min } / k_{B} T\right)^{z+1}} \int_{0}^{y_{\max }} y^{z} \exp (-y) d y,
\end{gathered}
$$

with $y_{\max }=\left(E_{\max }-E_{\min }\right) / k_{B} T$. Noting that $\Gamma(z+1, x)=\int_{0}^{x} y^{z} \exp (-y) d y$ is the second incomplete gamma function, Eq. (8) can be written as [13]:

$$
n_{D B}(T)=\omega_{D B} \tau_{D B}^{0} \frac{\exp \left(-E_{\min } / k_{B} T\right)}{\left(E_{\min } / k_{B} T\right)^{z+1}} \Gamma\left(z+1, \frac{E_{\max }-E_{\min }}{k_{B} T}\right) .
$$

It can be seen that the mean DB energy is higher than the averaged energy density (or temperature): 


$$
\left\langle E_{D B}\right\rangle=\frac{\int_{\min }^{E_{\max }} C_{D B}(E, T) E d E}{\int_{E_{\min }}^{E_{\max }} C_{D B}(E, T) d E} \underset{E_{\max } \gg E_{\min }}{\longrightarrow}\left(\frac{E_{\min }}{k_{B} T}+z+1\right) \times k_{B} T .
$$

Assuming, according to [22] that $E_{\min } / k_{B} T \approx 3$ and $\left\langle E_{D B}\right\rangle \approx 5 k_{B} T$, one obtains an estimate for $z \approx 1$, which corresponds to linear increase of the DB lifetime with energy.

\subsection{External driving}

Fluctuation activated nature of DB creation can be described in the framework of classical Kramers model, which is archetypal for investigations in reaction-rate theory [16]. The model considers a Brownian particle moving in a symmetric doublewell potential $U(x)$ (Fig. 6(a)). The particle is subject to fluctuational forces that are, for example, induced by coupling to a heat bath The fluctuational forces cause transitions between the neighboring potential wells with a rate given by the famous Kramers rate:

$$
\dot{R}_{K}\left(E_{0}, T\right)=\omega_{0} \exp \left(-E_{0} / k_{B} T\right),
$$

where $\omega_{0}$ is the attempt frequency and $E_{0}$ is the height of the potential barrier separating the two stable states, which, in the case of fluctuational DB creation, corresponds to the minimum energy that should be transferred to particular atoms in order to initiate a stable DB. Thus, the DB creation rate (3) is given by the Kramers rate: $K_{D B}(E, T)=\dot{R}_{K}(E, T)$.

In the presence of periodic modulation (driving) of the well depth (or the reaction barrier height) such as $U(x, t)=U(x)-V\left(x / x_{m}\right) \cos (\Omega t)$, the reaction $\dot{R}_{K}$ rate averaged over times exceeding the modulation period has been shown to increase according to the following equation [13]:

$$
\langle\dot{R}\rangle_{m}=\dot{R}_{K} I_{0}\left(\frac{V}{k_{B} T}\right),
$$

where the amplification factor $I_{0}(x)$ is the zero order modified Bessel function of the first kind. Note that the amplification factor is determined by the ratio of the modulation amplitude $V$ to temperature, and it does not depend on the modulation frequency or the mean barrier height. Thus, although the periodic forcing may be too weak to induce athermal reaction (if $V<E_{0}$ ), it can amplify the average reaction rate drastically if the ratio $V / k_{B} T$ is high enough, as it is demonstrated in Fig. 6(b). Another mechanism of enhancing the DB creation rate is based on small stochastic modulations of the DB activation barriers caused by external driving. Stochastic driving has been shown to enhance the reaction rates via effective reduction of the 


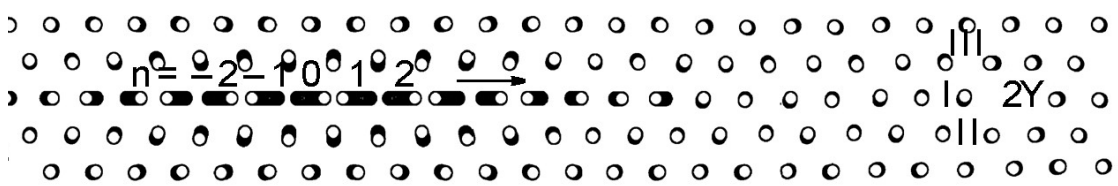

Fig. 7 Illustration of moving DB (quodon) before "collision" with a vacancy in 2D crystal (4 times zoom of atomic displacements) [26]. $2 Y$ is the distance between the atoms II and III. Reproduced with permission from Kistanov, A., Dmitriev, S., Semenov, A.S., Dubinko, V., Terentyev, D.: Interaction of propagating discrete breathers with a vacancy in a two-dimensional crystal. Tech. Phys. Lett. 40, 657661 (2014). Copyright (2014) Springer.

underlying reaction barriers $[10,12]$ as:

$$
\langle\dot{R}\rangle=\omega_{0} \exp \left(-E_{a}^{D B} / k_{B} T\right), \quad E_{a}^{D B}=E_{0}-\frac{\langle V\rangle_{S D}^{2}}{2 k_{B} T}, \quad \text { if }\langle V\rangle_{S D} \ll k_{B} T,
$$

where $\langle V\rangle_{S D}$ is the standard deviation of the potential energy of atoms surrounding the activation site.

In the present view, the DB creation is seen as a chemical reaction activated by thermally or externally induced fluctuations. In the following section we consider the reaction of annealing of defects in crystals, such as the deep traps for electrons/holes, within the similar framework. I simplified model can be seen in Ref. [8]

\section{Amplification of Sb-vacancy annealing rate in germanium by DBs}

Sb-vacancy defect in Ge is a typical deep trap, which has been shown to arise under displacement damage (producing vacancies) and anneal either thermally (above $400 \mathrm{~K}$ ) or under ICP treatment at ambient temperatures of about $300 \mathrm{~K}$ [1]. This plasma-induced acceleration of annealing at depth extending up to several microns must be driven by some mechanism capable of transferring the excitation energy of surface atoms (interacting with plasma) deep into the crystal. Quodons are thought to be good candidates for providing such a mechanism, and bellow we present a model of quodon-enhanced defect annealing based on quasi-periodic modulation of the annealing activation barrier caused by the interaction of defects with a 'quodon gas'. This mechanism is illustrated in Fig. 7, which shows a moving DB (quodon) before 'collision' with a vacancy in 2D close-packed crystal with pairwise Morse interatomic potentials [26]. The DB velocity can be varied by changing the phase difference, $\delta$. The distance between the atoms II and III is $2 Y$ and $\Delta Y=Y-Y_{0}$ is the difference between the excited and ground state due to the interaction with a quodon, which is shown in Fig. 8 as a function of time for 'slow' and 'fast' DBs. The mean difference $\langle\Delta Y\rangle$ and standard deviation $\langle\Delta Y\rangle^{2}$ over the excitation time 

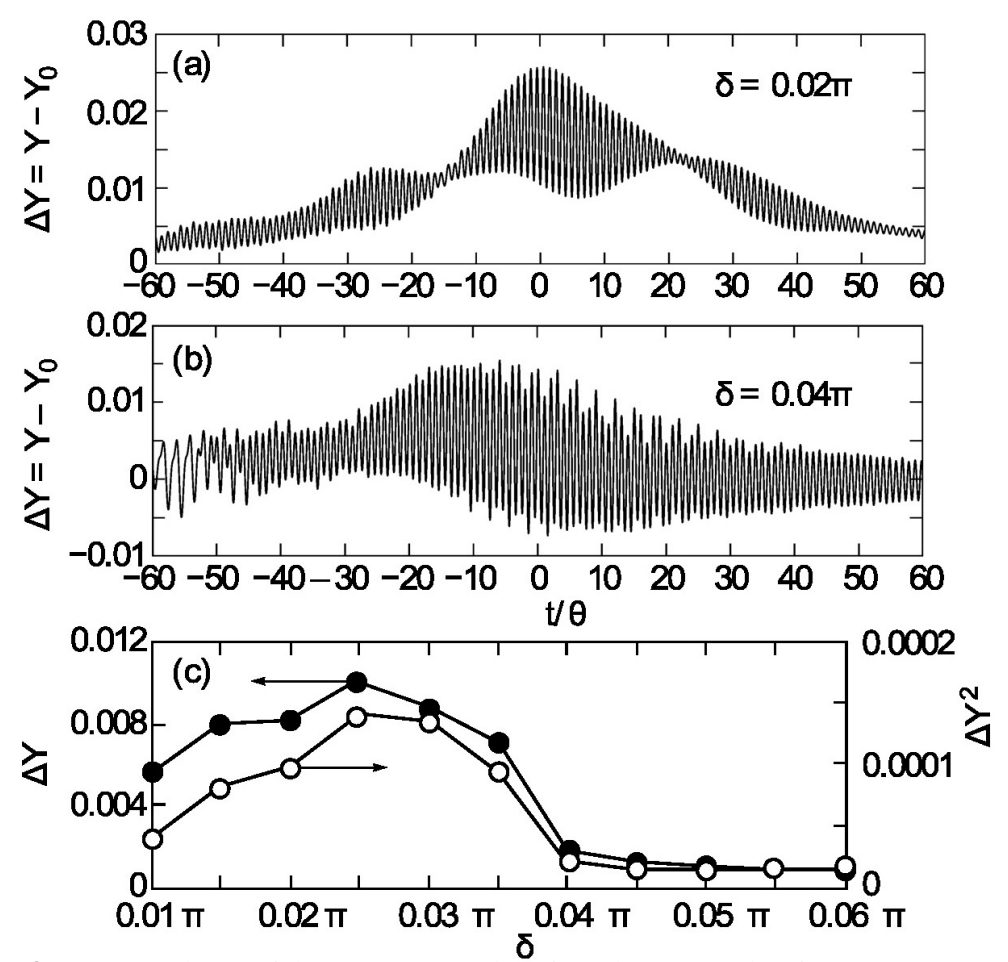

Fig. 8 (a) Dependence of $\Delta Y=Y-Y_{0}$ on time for "slow" DBs (b) "fast" DBs ; (c) Mean difference $\langle\Delta Y\rangle$ and standard deviation over 80 oscillation periods vs. phase difference $\langle\Delta Y\rangle^{2}$, which is proportional to the DB velocity. It can be seen that "slow" DBs disturb the vacancy more strongly than the "fast" ones [26]. Reproduced with permission from Kistanov, A., Dmitriev, S., Semenov, A.S., Dubinko, V., Terentyev, D.: Interaction of propagating discrete breathers with a vacancy in a two-dimensional crystal. Tech. Phys. Lett. 40, 657661 (2014). Copyright (2014) Springer.

of $\sim 80$ oscillation periods have been calculated. It can be seen that "slow" DBs disturb the vacancy more strongly than the "fast" ones, and besides, they practically do not lose their energy in the course of 'collision'. So these DBs behave similar to molecules of some gas, which can be 'pumped' from the surface into material up to some depth equal to the propagation range of quodons before the decay. Then, the average rate of quodon generation (per atom), will be proportional to the ratio of their flux $\Phi_{q}$ though the surface (where they are created by energetic plasma atoms) to the propagation range of quodons, $l_{q}$ :

$$
K_{q}=\frac{\Phi_{q}}{l_{q}} \omega_{\mathrm{Ge}}, \quad \Phi_{q}=\Phi_{\mathrm{Ar}} \frac{4 E_{\mathrm{Ar}} M_{\mathrm{Ar}} M_{\mathrm{Ge}}}{E_{q}\left(M_{\mathrm{Ar}}+M_{\mathrm{Ge}}\right)^{2}},
$$

where $\omega_{\mathrm{Ge}}$ is the $\mathrm{Ge}$ atomic volume, $M_{\mathrm{Ar}}, M_{\mathrm{Ge}}$ are the $\mathrm{Ar}$ and $\mathrm{Ge}$ atomic masses, $\Phi_{\mathrm{Ar}}$ is the flux of Ar ions or atoms with a mean energy $E_{\mathrm{Ar}}$, a part of which 


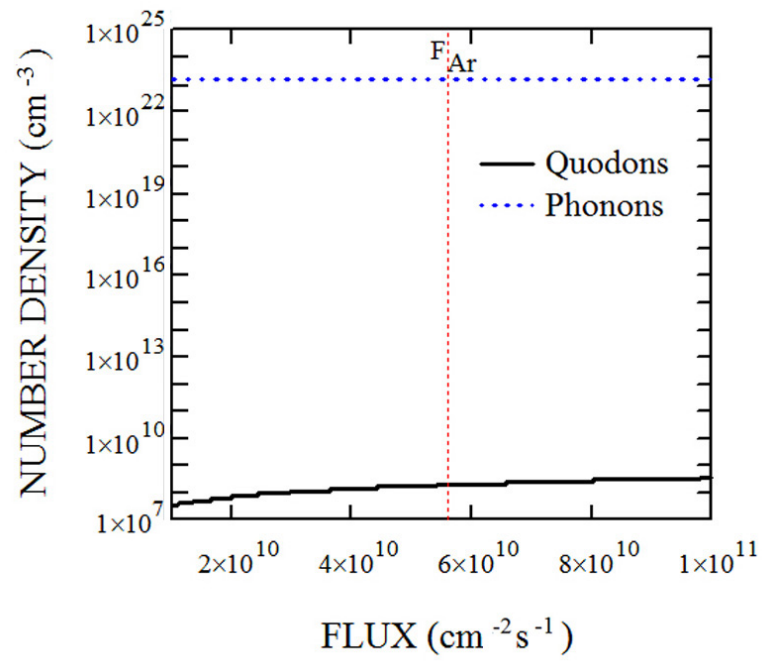

Fig. 9 The density of quodon gas, $C_{q} / \omega_{G e}$, vs. Ar flux at the irradiation temperature of $300 \mathrm{~K}$ within the layer of thickness $L_{q}=5.3$ microns, at the quodon velocity of $v_{q}=300 \mathrm{~m} / \mathrm{s}$. Density of the phonons at $300 \mathrm{~K}$ is shown for comparison with a dotted line. The vertical dotted line corresponds to Ar flux in the experiment [1].

$4 M_{\mathrm{Ar}} M_{\mathrm{Ge}} /\left(M_{\mathrm{Ar}}+M_{\mathrm{Ge}}\right)^{2}$, is transferred to germanium atoms and could be spent on the generation of quodons with a mean energy $E_{q}$. Then the steady-state concentration of quodon gas (see Fig. 9) will be given simply by the product of their generation rate and the life-time, $\tau_{q}$ :

$$
C_{q}=K_{q} \tau_{q}, \quad \tau_{q}=\frac{l_{q}}{v_{q}}, \text { then } \quad C_{q}=\frac{\Phi_{q} \omega_{\mathrm{Ge}}}{v_{q}},
$$

where $v_{q}$ is the quodon propagation speed, which actually determines their concentration within the layer of a thickness $l_{q}$ (Fig. 9).

Consider the periodic modulation of the defect annealing activation energy in more details. It is driven by quodons that scatter on the defects and excite the surrounding atoms (Fig. 8). The amplitude of the quasi-periodic energy deviation $V_{e x}$ can be in the $\mathrm{eV}$ range with the excitation time, $\tau_{e x}$, of about 100 oscillation periods. In the modified Kramers model (12), this energy deviation corresponds to the modulation of the annealing activation barrier. Then, a macroscopic annealing rate (per defect per second) may be written as follows:

$$
\langle\dot{R}\rangle_{\text {macro }}=\omega_{0} \exp \left(-\frac{E_{a}}{k_{B} T}\right)\left(1+\left\langle I_{0} \frac{V_{e x}}{k_{B} T}\right\rangle \omega_{e x} \tau_{e x}\right),
$$

where $E_{a}$ is the annealing activation energy, $\omega_{e x}$ is the mean number of excitations per defect per second caused by the flux of quodons, which is proportional to the 

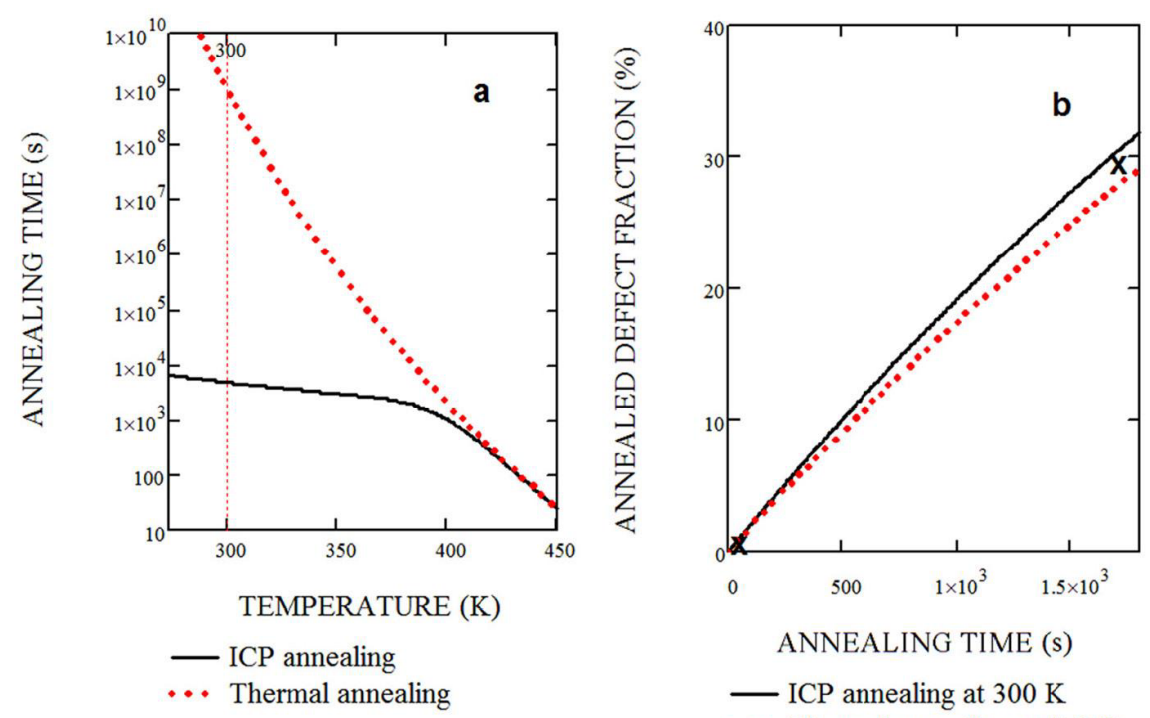

ANNEALING TIME (s)

ICP annealing at $300 \mathrm{~K}$

Fig. 10 (a) Characteristic annealing time, Eq. (19) under thermal thermal annealing at $373 \mathrm{~K}$ defect fraction with time during thermal annealing at $373 \mathrm{~K}$ in comparison with ICP-induced annealing at $300 \mathrm{~K}$ according to the Eq. (18) and experimental data X. Irradiation and material parameters: $F_{A r}=5.6 \times 10^{10} \mathrm{~cm}^{-2} \mathrm{~s}^{-1} ; \tau_{e x}=10^{-11} \mathrm{~s} ; \omega_{0}=5.313 \times 10^{13} \mathrm{~s}^{-1} ; E_{a}=1.35 \mathrm{eV} ; V_{e x}=1.28$ $\mathrm{eV}$.

quodon flux and the cross-section of quodon-defect interaction and is given by

$$
\omega_{e x}=\Phi_{q} \pi b^{2}
$$

where $b$ is the atomic spacing, the quodon formation energy $V_{q} \approx V_{e x}$. For material parameters presented in Fig. 10, one has $\omega_{e x} \approx 10^{-4} \mathrm{~s}^{-1}$.

$\mathrm{Sb}$-vacancy annealing kinetics is described by the following equation for the defect concentration:

$$
\frac{d c_{d}}{d t}=-\frac{c_{d}}{\tau_{a}}, \quad c_{d}(t)=c_{d}(0) \exp \left(-\frac{t}{\tau_{a}}\right),
$$

where $\tau_{a}$ is the characteristic annealing time, which inversely proportional to the annealing reaction rate given by Eq. (16)

$$
\tau_{a}=\frac{\exp \left(\frac{E_{a}}{k_{B} T}\right)}{\omega_{0}\left(1+I_{0}\left(\frac{E_{e x}}{k_{B} T}\right) \omega_{e x} \tau_{e x}\right)} .
$$

In the absence of driving ( $\Phi_{\mathrm{Ar}}=0=>\omega_{e x}=0$ ), Eq. (19) describes the thermal annealing, while at $\Phi_{\mathrm{Ar}}>0$, the annealing proceeds at room temperatures at a rate which is 5 orders of magnitude higher than that at $\Phi_{\mathrm{Ar}}=0$, and it is comparable to 


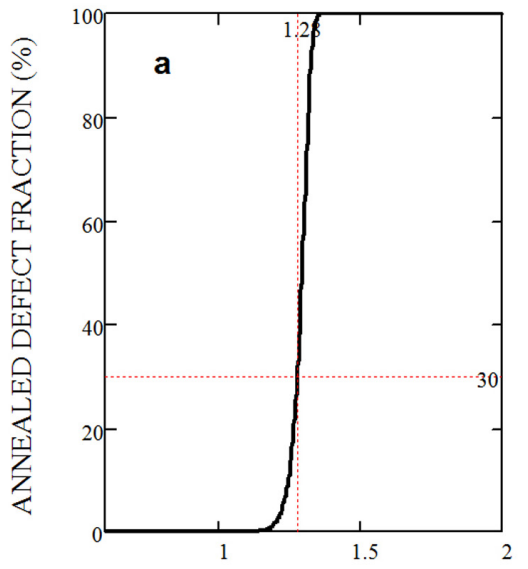

EXCITATION ENERGY $(\mathrm{eV})$

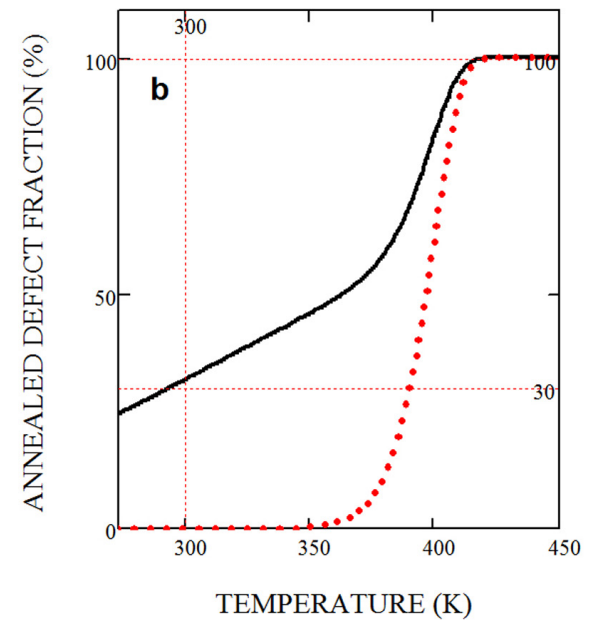

Fig. 11 (a) Annealed defect fraction at after $30 \mathrm{~min}$ of ICP vs. excitation energy, $V_{e x}$, at $300 \mathrm{~K}$; (b) after $30 \mathrm{~min}$ of ICP or heating vs. temperature at $V_{e x}=1.28 \mathrm{eV}$.

the thermal annealing at the boiling point $(373 \mathrm{~K})$, as demonstrated in Fig. 10. In agreement with experimental data [1], the defect concentration decreases by $30 \%$ after ICP treatment for $30 \mathrm{~min}$ at room temperature.

The ICP-annealing rate is very sensitive to the excitation energy (Fig. 11(a)), and it increases monotonously with temperature (Fig. 11(b)), provided that the quodon production rate and propagation range are temperature independent.

\section{Summary}

A new mechanism of the long-range annealing of defects in Ge under low energy ICP treatment is proposed, which is based on the catalyzing effect of DBs on annealing reactions. The moving DB (quodon) creation is triggered by Ar flux which provides the input energy transformed into the lattice vibrations.

Simple analytical expressions for the annealing rate under ICP treatment are derived as functions of temperature, ion current and material parameters, which show a good agreement with experimental data. 


\section{Acknowledgments}

S.V.D. thanks the Tomsk State University Academic D.I. Mendeleev Fund Program.

\section{References}

1. Archilla, J.F.R., Coelho, S.M.M., Auret, F.D., Dubinko, V.I., Hizhnyakov, V.: Long range annealing of defects in germanium by low energy plasma ions. Physica D 297, 56-61 (2015)

2. Archilla, J.F.R., Coelho, S.M.M., Auret, F.D., Nyamhere, C., Dubinko, V.I., Hizhnyakov, V.: Experimental observation of moving intrinsic localized modes in germanium. In: J.F.R. Archilla, N. Jiménez, V.J. Sánchez-Morcillo, L.M. García-Raffi (eds.) Quodons in mica: nonlinear localized travelling excitations in crystals. Springer (2015). To appear

3. Archilla, J.F.R., Cuevas, J., Alba, M.D., Naranjo, M., Trillo, J.M.: Discrete breathers for understanding reconstructive mineral processes at low temperatures. J. Phys. Chem. B 110(47), 24,112 (2006)

4. Auret, F.D., Coelho, S., Myburg, G., van Rensburg, P.J.J., Meyer, W.E.: Defect introduction in Ge during inductively coupled plasma etching and schottky barrier diode fabrication processes. Thin Solid Films 518(9), 2485-2488 (2010)

5. Auret, F.D., van Rensburg, P.J.J., Hayes, M., Nel, J.M., Coelho, S., Meyer, W.E., Decoster, S., Matias, V., Vantomme, A., Smeets, D.: Electrical characterization of defects in heavy-ion implanted n-type Ge. Nucl. Instrum. Meth. B 257(1-2), 169-171 (2007)

6. Baimova, J.A., Dmitriev, S.V., Zhou, K.: Discrete breather clusters in strained graphene. Europhys. Lett. 100, 36,005 (2012)

7. Chechin, G.M., Dmitriev, S.V., Lobzenko, I.P., Ryabov, D.S.: Properties of discrete breathers in graphane from ab initio simulations. Phys. Rev. B 90, 045,432 (2014)

8. Coelho, S.M.M., Archilla, J.F.R., Auret, F.D., Nel, J.M.: The origin of defects induced in ultrapure germanium by electron beam deposition. In: J.F.R. Archilla, N. Jiménez, V.J. SánchezMorcillo, L.M. García-Raffi (eds.) Quodons in mica: nonlinear localized travelling excitations in crystals. Springer (2015). To appear

9. Dubinko, V., Russell, F.: Radiation damage and recovery due to the interaction of crystal defects with anharmonic lattice excitations. J. Nucl. Mater. 419, 378-385 (2011)

10. Dubinko, V., Shapovalov, R.: Theory of a quodon gas. with application to precipitation kinetics in solids under irradiation. In: R. Carretero-González, et al. (eds.) Localized Excitations in Nonlinear Complex Systems, vol. 7, pp. 265-288. Springer, New York (2014)

11. Dubinko, V.I.: Low-energy nuclear reactions driven by discrete breathers. J. Condensed Matter Nucl. Sci. 14 (2014)

12. Dubinko, V.I., Dubinko, A.V.: Modification of reaction rates under irradiation of crystalline solids: Contribution from intrinsic localized modes. Nucl. Instrum. Meth. B 303, 133-135 (2013)

13. Dubinko, V.I., Selyshchev, P.A., Archilla, J.F.R.: Reaction-rate theory with account of the crystal anharmonicity. Phys. Rev. E 83, 041,124 (2011)

14. Flach, S., Gorbach, A.V.: Discrete breathers advances in theory and applications. Phys. Rep. 467, 1-116 (2008)

15. Haas, M., Hizhnyakov, V., Shelkan, A., Klopov, M., Sievers, A.J.: Prediction of highfrequency intrinsic localized modes in Ni and Nb. Phys. Rev. B 84, 144,303 (2011)

16. Hanggi, P., Talkner, P., Borkovec, M.: Reaction-rate theory: fifty years after Kramers. Rev. Mod. Phys. 62, 251-341 (1990)

17. Hizhnyakov, V.: Relaxation jumps of strong vibration. Phys. Rev. B 53, 13,981-13,984 (1996) 
18. Hizhnyakov, V., Haas, M., Pishtshev, A., Shelkan, A., Klopov, M.: Theory and molecular dynamics simulations of intrinsic localized modes and defect formation in solids. Phys. Scr. 89(4), 044,003 (2014)

19. Hizhnyakov, V., Haas, M., Shelkan, A., Klopov, M.: Standing and moving discrete breathers with frequencies above the phonon spectrum. In: J.F.R. Archilla, N. Jiménez, V.J. SánchezMorcillo, L.M. García-Raffi (eds.) Quodons in mica: nonlinear localized travelling excitations in crystals. Springer (2015). To appear

20. Hizhnyakov, V., Nevedrov, D., Sievers, A.J.: Quantum properties of intrinsic localized modes. Physica B 316-317, 132-135 (2002)

21. Khadeeva, L.Z., Dmitriev, S.V.: Discrete breathers in crystals with $\mathrm{NaCl}$ structure. Phys. Rev. B 81, 214,306 (2010)

22. Khadeeva, L.Z., Dmitriev, S.V.: Lifetime of gap discrete breathers in diatomic crystals at thermal equilibrium. Phys. Rev. B 84, 144,304 (2011)

23. Khadeeva, L.Z., Dmitriev, S.V., Kivshar, Y.S.: Discrete breathers in deformed graphene. JETP Lett. 94, 539 (2011)

24. Kiselev, S.A., Bickham, S., Sievers, A.J.: Anharmonic gap modes in a perfect one-dimensional diatomic lattice for standard two-body nearest-neighbor potentials. Phys. Rev. B 48, 13,508 (1993)

25. Kiselev, S.A., Sievers, A.J.: Generation of intrinsic vibrational gap modes in three-dimensional ionic crystals. Phys. Rev. B 55, 5755 (1997)

26. Kistanov, A., Dmitriev, S., Semenov, A.S., Dubinko, V., Terentyev, D.: Interaction of propagating discrete breathers with a vacancy in a two-dimensional crystal. Tech. Phys. Lett. 40, 657661 (2014)

27. Korznikova, E., Baimova, J.A., Dmitriev, S.V.: Effect of strain on gap discrete breathers at the edge of armchair graphene nanoribbons. Europhys. Lett. 102, 60,004 (2013)

28. Liu, B., Baimova, J.A., Dmitriev, S.V., Wang, X., Zhu, H., Zhou, K.: Discrete breathers in hydrogenated graphene. J. Phys. D: Appl. Phys. 46, 305,302 (2013)

29. Manley, M.E.: Impact of intrinsic localized modes of atomic motion on materials properties. Acta Mater. 58, 2926-2935 (2010)

30. Markevich, V.P., Peaker, A.R., Litvinov, V.V., Emtsev, V.V., Murin, L.I.: Electronic properties of antimony-vacancy complex in Ge crystals. J. Appl. Phys. 95(8), 4078-4083 (2004)

31. Medvedev, N.N., Starostenkov, M.D., Manley, M.E.: Energy localization on the Al sublattice of $\mathrm{Pt}_{3} \mathrm{Al}$ with $\mathrm{L}_{2}$ order. J. Appl. Phys. 114, 213,506 (2013)

32. Murzaev, R.T., Kistanov, A.A., Dubinko, V.I., Terentyev, D.A., Dmitriev, S.V.: Moving discrete breathers in bcc metals V, Fe and W. Comput. Mater. Sci. 98, 88 (2015)

33. Piazza, F., Lepri, S., Livi, R.: Cooling nonlinear lattices toward energy localization. Chaos 13, 637-645 (2003)

34. Russell, F.M., Eilbeck, J.C.: Evidence for moving breathers in a layered crystal insulator at 300 K. Europhys. Lett. 78, 10,004-10,012 (2007)

35. Sievers, A.J., Takeno, S.: Intrinsic localized modes in anharmonic crystals. Phys. Rev. Lett. 61, 970-973 (1988)

36. Terentyev, D., Dubinko, A., Dubinko, V., Dmitriev, S., Zhurkin, E.: Interaction of discrete breathers with primary lattice defects in bcc Fe. Submitted

37. Voulgarakis, N., Hadjisavvas, G., Kelires, P., Tsironis, G.: Computational investigation of intrinsic localization in crystalline Si. Phys. Rev. B 69, 113,201 (2004) 